\title{
Adolescent psychoses: treatment and service provision
}

\author{
W Ll Parry-Jones
}

The generic term 'psychosis' is enduring but imprecise and the traditional division between neurosis and psychosis will be discouraged in the 10th revision of the International Classification of Diseases (ICD-10), to be published in 1992. ${ }^{1}$ However, it is used in ICD- $9^{2}$ and in the Diagnostic and Statistical Manual of Mental Disorders (DSM-III-R). ${ }^{3}$ It still has an established place in psychiatric parlance, indicating the presence of hallucinations, delusions or certain types of abnormal behaviour, namely, gross excitement and over-activity; severe, prolonged, social withdrawal; marked psychomotor retardation and catatonic behaviour. Its use does not imply either greater severity, although this is often the case, or impairment of insight or reality testing. With these reservations, it is permissible to refer to a group of adolescent psychoses. Categories of psychoses in ICD-9 include those with origin specific to childhood, principally infantile autism, but this condition is viewed currently as a pervasive developmental disorder and no longer a form of childhood psychosis. This review is concerned chiefly with schizophrenia, schizotypal disorders, drug induced psychoses, and affective disorders arising in adolescence.

There is no unique adolescent psychosis and the view that 'adolescent turmoil' represents a 'normal psychosis' is outdated. ${ }^{4}$ Instead, adolescent disorders need to be seen in the context of all psychoses occurring in children and adults, and it is generally accepted that adult diagnostic criteria are applicable. Nevertheless, adolescence has a pathoplastic influence, giving disorders staged characteristics and colouring the content of psychotic symptomatology, sometimes making recognition difficult.

Morbidity surveys suggest that the one year prevalence of psychosis in mid-adolescence is less than one per thousand. ${ }^{5}$ The proportion of psychotic patients in adolescent outpatient clinics is commonly about $5 \%$ of referrals ${ }^{6}$ and reported figures for inpatients vary from $2 \%$ to over $22 \%$. About $3 \%$ of all psychiatric first admissions for children aged $10-14$ years are for psychoses, but the figure rises to $21 \%$ for those aged 15-19 years.

The psychoses occupy a neglected place in adolescent medicine and psychiatry. Services for psychotic adolescents are not well developed and academic interest has been limited. This is explained partly by the fact that the prominent interests of staff drawn to work with adolescents are in maturational, social, and family processes. Disorders construed in terms of disturbance of these processes suit multidisciplinary team consensus, as the emergent treatments are interdisciplinary, for example, family therapy. Psychotic disorders, traditionally the prerogative of doctors and nurses, however, have not fitted readily into this social dynamic model. The unknown aetiology, with genetic and possible organic factors, precludes developmental or psychodynamic explanations and pharmacotherapy tends to exclude non-medical staff. Interest in psychosis may be diluted also if staff have little or no formal psychiatric training or experience of adult disorders, because the incomprehensibility of psychosis can be alienating.

\section{Aetiology}

The psychoses comprise a disparate group of disorders and there is no single aetiological pattern. There are well established genetic factors in schizophrenia and the affective disorders. While there may be childhood precufsors of adult schizophrenia, these are not sufficiently specific to enable early detection. Some psychotic reactions may be symptomatic of organic disorders, but neurological and biochemical leads in schizophrenia and the affective disorders have so far proved inconclusive. Stressful life events often play a part in precipitating disorders and exposure to high levels of emotion in the family promotes relapse in schizophrenia. Despite much speculation, there is no enduring evidence for the causative importance of abnormal family processes, although family factors may play a part in either maintaining or abbreviating psychotic episodes.

\section{Diagnosis}

Presenting problems are likely to be very varied, including violence, destructive outbursts, bizarre behaviour, delusional states, suicide attempts, excitement, disinhibited sexual behaviour, and repeated absconding. As psychotic symptomatology displays such diversity, careful syndromal diagnosis is essential for clinical management, because of the different treatment strategies that may be indicated. A key task is disentangling maturational features, such as emotional lability and ordinary adolescent concerns, from psycho- 
pathological phenomena. Adolescents with severe disturbance of emotional and personality development (borderline disorders) are hard to understand and may appear incoherent, but very few have psychotic disorders. ${ }^{8}$ It is characteristic of the adolescent psychoses, however, that precise diagnosis may be difficult and lengthy observation and assessment may be necessary. This applies particularly to distinguishing between first episodes of schizophrenia and mania, but such difficulty should encourage clinicians to be as rigorous as possible in diagnosis and not simply to resort to the unqualified use of the term adolescent psychosis. In all cases, possible organic causes of psychosis, including rare neurodegenerative conditions, need to be investigated. Clarification of the psychiatric syndrome only forms one part of the full diagnostic formulation, which comprises consideration of specific developmental delays, intellectual level, medical conditions, and abnormal psychosocial situations.

Identification of organic psychosis due, for example, to toxic states, is likely to be clear cut, although the precise causation can be obscure. However, differential diagnosis can be difficult when there is the possibility of organic brain disease. For example, the early signs of subacute sclerosing panencephalitis may resemble schizophrenia, until progressive dementia, with myoclonus and epilepsy, supervenes. Seizure disorder, especially when there is temporal lobe involvement, can be associated with schizophrenia-like psychosis, and anticonvulsants themselves can also produce psychotic states. Drug induced disorders will be influenced by the substance involved, for example, cocaine, hallucinogens, or cannabis, and are characterised usually by hallucinations, delusions, misidentifications, psychomotor disturbance, and abnormal affect. Occasionally, hallucinations can be induced by prescribed drugs (for example, methylphenidate, promethazine hydrochloride, and pseudoephedrine). Schizophrenia is rare in childhood and knowledge regarding it remains limited. ${ }^{9-11}$ Incidence increases after puberty and the usual age of onset is 15 to 45 years. In the initial clinical picture of adolescent schizophrenia, Schneider's first rank symptoms may be fleeting, difficult to elicit, or absent. ${ }^{12}$ The common presentation is the acute syndrome, with falling off in social and academic performance, mood disturbance, incoherent speech, bizarre actions, hallucinations, delusions, and preoccupation with inner thoughts. Diagnostic problems arise if there are affective features, insidious deterioration of personality, or evidence of clouding of consciousness and perplexity. It is not uncommon for apparently affective disorders to declare themselves eventually as schizophrenic. Paranoid states, without typical schizophrenic features, are unusual in adolescents. Reactive (psychogenic) psychoses, attributable largely or entirely to stressful experiences may occur, but distinction from schizophrenia is uncertain. They are often transient and appear more likely to occur in teenagers from ethnic minorities.

Affective psychoses, characterised by mood swings to depression or elation, and cyclothymic symptoms are rare before puberty, but gradually increase in incidence during adolescence to reach adult levels. Although depressive features are common in adolescence, recognition of affective psychoses, resembling adult 'endogenous' syndromes, may be hampered by developmental differences in symptomatology. ${ }^{1314}$ Depression in adolescents is influenced by environment more readily than in adults; lowering of mood is less fixed and features like self depreciation are infrequent. Suicide is very rare before puberty, but incidence increases during adolescence. First episodes of adolescent mania may be misdiagnosed as schizophrenia $^{15}$ unless classic features are present but, after several relapses, a recurrent or cyclical pattern may emerge. There is a risk of misinterpreting symptoms like euphoria and being misled by potential psychogenic factors, which may mask and aggravate, but not necessarily cause, affective disorders.

\section{Treatment}

\section{SERVICE PROVISION}

Although the number of psychotic adolescents is small, they can make disproportionate demands on staff and resources. The service requirements are clear. Psychiatric outpatient and liaison services must be able to respond promptly to urgent referrals and there needs to be an accessible inpatient adolescent unit with emergency beds and a flexible admission policy so that, if required, hospitalisation can occur without delay. The unit milieu needs to be such that psychotic patients are not excluded and that it is conducive to eclectic treatment. Skilled, adaptable nursing is required, on a 24 hour basis, seven days a week. When adolescents present with possible psychotic states in paediatric or general medical settings, early involvement of an adolesscent psychiatrist is desirable, for diagnostic assessment and assistance in management, especially if continuing medical investigation is indicated. This also facilitates subsequent transfer to a psychiatric unit if necessary.

Despite the proliferation of psychiatric adolescent units since the late 1960 s there are still significant deficiencies. ${ }^{16}$ The Health Advisory Service found recently that clinical services were variable and incomplete, ${ }^{17}$ especially for acutely disturbed adolescents, emergencies, rehabilitation, and long term care. Treatment of psychotic teenagers may still occur in adult mental hospital wards and, although this need not be wholly adverse, the adolescent is likely to miss, at least, social and educational activities with peers. The optimum admission policy for adolescent units is controversial, the main distinction being between general purpose units, catering for a wide range of patients, and those concentrating on specific diagnostic groups or specialised therapeutic approaches, the latter models running the risk of excluding the psychoses.

INITIAL TREATMENT

The optimum management of psychotic 
adolescents calls for the earliest possible identification and diagnosis, although many child and adolescent psychiatrists are reticent about the implications of identifying severe, potentially life long disorders like schizophrenia in children and teenagers. Treatment will be concerned with a wider range of problems and handicaps than the psychotic process and intervention has to be tailored pragmatically to the symptomatology and needs of individual teenagers and their families.

Although the overall aim is to use outpatient or day hospital care as far as possible, hospital admission is commonly advisable, often after an emergency referral. Parental authority for admission is required for children under 16 years and, occasionally, compulsory admission under the Mental Health Act 1983, or the Mental Health (Scotland) Act 1984, is needed. The intention should be to employ hospitalisation only during the acute phase of the disorder, while assessment and diagnosis are undertaken, treatment initiated, plans for rehabilitation and aftercare are being established, and the coping capacity of parents and others involved in management is restored or enhanced.

Individual psychiatric treatment needs to be based on straightforward communication and counselling about day to day maturational, social, and educational problems. It should be supportive and non-confrontational and, for these reasons, exposure to stressful group therapy is inappropriate. There are few indications for psychoanalytic psychotherapy. There is always an important role for family work, aimed at providing candid information and support, reducing high expressed emotion in the families of schizophrenics and, in all cases, encouraging realistic expectations, the acceptance of relapses, and the possibility of long term disabilities. ${ }^{18}$

Pharmacotherapy is essential in most adolescent onset psychoses. Treatment with phenothiazines (for example, chlorpromazine), thioxanthenes (for example, flupenthixol), or butyrophenones (for example, haloperidol) is the mainstay in acute and chronic schizophrenia and schizotypal disorders. Depot preparations are particularly useful for maintenance treatment in adolescents who are unreliable in taking oral medication. Heterocyclic antidepressants and newer unrelated preparations, such as fluoxetine, can be used in adolescent depression, but there is limited information about their efficacy and documented recommendations are not available. ${ }^{19}$ Occasional prophylactic use is made of lithium carbonate. ${ }^{20}$ Electroconvulsive therapy is rarely necessary with adolescents, but may be indicated in severe protracted depression.

There is considerable scope for behavioural modification, particularly in reducing withdrawn or socially unacceptable behaviour. Psychotic adolescents should be encouraged to continue their usual educational instruction and social and recreational activities, supplemented by occupational therapy, social skills training, and enhancement of independent self help skills.

REHABILITATION AND LONG TERM CARE

After initial assessment and treatment or discharge from hospital, long term psychiatric care and recurrent hospitalisation are often required. For example, schizophrenic patients will need to continue medication for several months. Most patients are likely to live at home, but there is limited provision of supervised hostel accommodation, group homes, and residential treatment centres for those unable to live with their families. Appropriate educational provisions may be hard to find, especially on a residential basis, although there are increasing facilities for young people with special needs in colleges of further education. Similarly, difficulties may arise in preparing for or re-entering employment. In general, facilities for rehabilitation and long term care are unsatisfactory. Overlap with adult psychiatric services is often inadequate and unplanned and the special needs of chronically mentally ill adolescents and young adults may be overlooked.

\section{MANAGEMENT ISSUES CAUSED BY PSYCHOTIC ADOLESCENTS}

Problems arising in the inpatient management of psychotic adolescents, particularly in general purpose psychiatric units, need to be understood by all adolescent health care personnel. They illustrate those problems that are generated in any treatment setting where there is a need to integrate and treat a few odd, sometimes frightening, young people with others, whose disorders are more comprehensible and readily treatable.

Differing staff attitudes towards the concept of psychosis can create difficulties in constructing realistic treatment objectives. Even diagnosis may be emotive because of fears about 'labelling' devaluing the patient and reducing treatment to empirical medication. While outside hospital preconceived ideas about such diagnostic labels as schizophrenia can be unfavourable, staff misapprehensions about the clinical value of diagnosis have to be resolved. In the psychoses in particular, patient care needs the benefit of all available knowledge about course and prognosis. Diagnostic formulations that deflect attention from the possible seriousness of disorder do patients and relatives a dis-service. ${ }^{21}$ Lack of a working diagnosis compounds the difficulties of coping with clinical ambiguity, leading to uncertainty about therapeutic objectives and loss of direction in management. Inconsistent staff responses follow, heightening confusion for patients and parents.

Division of opinion in the treatment team may concern how far psychotic behaviour can be controlled by the individual patient and the applicability of ordinary, age appropriate expectations of teenagers. In this respect, it is crucial to prevent the isolation of psychotic adolescents from their peers and to face them with expectations that are compatible with their prevailing level of function. The use of psychotropic drugs may be controversial if medication is construed simply as an expedient way of achieving control, without understanding the problem. The form and consistency of staff responses needs consideration. Grossly regressed behaviour, for example, can generate irritation, 
rejection, and even a punitive approach. Although an overtly confused, dependent adolescent may evoke caring, protective feelings, difficulties may arise in responding to aggressive, unpredictable, and inaccessible patients. Such young people require close involvement, but impaired contact with reality may be alienating to inexperienced staff and it is helpful if senior staff have had experience with adult psychotic patients.

Consideration needs to be given to the effect on the psychotic patient of being in a busy, high turnover unit, where most patients expect a short stay. The effects of a long stay in this setting can be adverse and it may be impossible to create the stable atmosphere conducive to rehabilitation for the patients who need extended treatment. Reactions of the main group of adolescents to their psychotic peers cannot be overlooked, as strange behaviour may be frightening and evoke rejection or teasing. Invariably, however, problems settle after explanation and reassurance. The essential 'normality' of other patients and the mutual acceptance that usually prevails are valuable therapeutically for psychotic patients. Occasionally, complaints and fears are expressed by parents of nonpsychotic patients.

\section{Prognosis}

Even after prompt, vigorous treatment most adolescent psychotics are likely to display a chronic, fluctuating course. Length of stay in hospital is likely to be longer than for other diagnostic groups. ${ }^{22}$ This is particularly the case in schizophrenia and allied disorders, which may progress to a chronic stage characterised by negative symptoms, such as apathy, paucity of speech, and blunting of affect. In schizophrenia, favourable prognostic pointers include acute onset, associated acute stress, affective features, higher intelligence, and good premorbid personality. In general, the younger the onset, the worse the prognosis. ${ }^{23}$ Adolescents with affective disorder are at risk for further episodes, especially if they displayed a bipolar or psychotic clinical picture.

\section{Conclusions}

Adolescent psychoses make up a small, but challenging, part of the work of adolescent psychiatric services, which can be overshadowed easily by the bulk of the work with less severe forms of disturbance. Psychotic disorders can be managed effectively in mixed, short stay general purpose psychiatric units, without impairing the treatment of other patients, provided the operational policy is planned for their reception. All staff in adolescent services should be able to cope with the ambiguity created by the uncertain causation of the psychoses, the need for empirical treatment, and the prospect of poor outcome. Early identification and diagnosis is essential and prognostic pointers need to be taken seriously. Overoptimism about outcome, because adolescence is a time of change, can be misplaced.

Adolescent services should give priority to the management of psychotic disorders and no adolescents, under the age of $\mathbf{1 6}$ years at least, should be admitted to adult mental hospitals because of lack of facilities or unduly selective admission policies of available adolescent units. Adolescents who relapse or deteriorate insidiously and are destined to become 'new chronic' patients need special attention, in hospital and the community, because existing continuing care and rehabilitation facilities are inadequate. Research priorities include both prospective studies to clarify the natural history and to establish predictors of outcome and the investigation of precursors of adult onset disorders.

1 World Health Organisation. ICD-10. 1989 Draft of chapter V. Mental and behavioural disorders. Clinical descriptions and diagnostic guidelines. Geneva: WHO, 1989.

2 World Health Organisation. Mental disorders: glossary and guide to their classification in accordance with the ninth revision of the international classification of diseases. Geneva: WHO 1978.

3 American Psychiatric Association. Diagnostic and statistical manual of mental disorders. 3rd Ed, revised. Washington, DC: American Psychiatric Association, 1987.

4 Rutter M, Graham P, Chadwick O, Yule W. Adolescent turmoil: fact or fiction? F Child Psychol Psychiatry 1976;17: 35-56.

5 Graham P, Rutter $M$. Adolescent disorders. In: Rutter $M$, Hersov L, eds. Child and adolescent psychiatry. Modern approaches. 2nd Ed. Oxford: Blackwell Scientific Publications, 1985:351-67.

6 Steinberg D, Galhenage DPC, Robinson SC. Two years' referrals to a regional adolescent unit: some implications for referrals to a regional adolescent unit: some implica

7 Department of Health and Social Security. Inpatient statistics from the mental health enquiry for England, 1982. London: HMSO, 1985.

8 Steinberg D. Psychotic and other severe disorders in adolescence. In: Rutter M, Hersov L, eds. Child and adolescent psychiatry. Modern approaches. 2nd Ed. Oxford: Blackwel Scientific Publications, 1985:567-83.

9 Kolvin I. Studies in the childhood psychoses. 1. Diagnostic criteria and classification. $B r \mathcal{F}$ Psychiatry 1971;118:381-4.

10 Eggers C. Psychoses in childhood and adolescence. Acto Paedopsychiatr 1982;48:81-98.

11 Volkmar FR, Cohen DJ, Hoshino Y, Rende RD, Paul R. Phenomenology and classification of childhood psychoses. Psychol Med 1988;18:191-201.

12 Garralda ME. Characteristics of the psychoses of late onset in children and adolescents (a comparative study of halchildren and adolescents (a comparative study
lucinating children). $\mathcal{F}$ Adolesc 1985;8:195-207.

13 Carlson G, Kashani J. Phenomenology of major depression from childhood through adulthood: analysis of three from childhood through adulthood: anaties. Am $\mathcal{F}$ Psychiatry 1988;145:1222-5.
studies

14 Parry-Jones WLI. Depression in adolescence. In: Herbst $\mathrm{K}$ Paykel E, eds. Depression: an integrative qpproach. Oxford:
Pater Heinemann, 1989:111-23.

15 Carlson GA. Child and 'adolescent mania-diagnostic considerations. I Child Psychol Psychiatry 1990;31:331-4

16 Parry-Jones WLI. Adolescent psychiatric services: Develop ment and expansion. In: Hendriks JH, Black M, eds. Child and adolescent psychiatry: into the 1990s. London: Royal College of Psychiatrists, 1990:83-9. (Occasional paper OP8.

17 Health Advisory Service. Bridges over troubled waters. London: HMSO, 1986

18 Atkinson JM. Schizophrenia at home. A guide to helping the family. London: Croom Helm, 1986.

19 Ryan ND. Heterocyclic antidepressants in children and adolescents. Fournal of Child and Adolescent Psychopharmacology 1990;1:21-31.

20 Steinberg $D$. The use of lithium carbonate in adolescence. f Child Psychol Psychiatry 1980;21:263-71.

21 Schulz SC, Koller MM. Schizophrenia and schizophreniform disorder. In: Hsu LKG, Hersen M, eds. Recent developdisorder. In: Hsu LKG, Hersen M, eds. Recent develop-
ments in adolescent psychiatry. New York: John Wiley, ments in adolesce
1989:289-308.

22 Pyne N, Morrison R, Ainsworth P. A follow-up study of the first 70 admissions to a general purpose adolescent unit. first 70 admissions to a
$\mathcal{f}$ Adolesc $1985 ; 8: 333-45$.

23 Kydd RR, Werry JS. Schizophrenia in children under sixteen years. F Autism Dev Disord 1982;12:343-57. 